\title{
Enhancing the resolution of seismic data based on the generalized S-transform
}

\author{
Tian Jianhua*, Song Wei and Yang Feizhou \\ State Key Laboratory of Petroleum Resource and Prospecting, China University of Petroleum, Beijing 102249, China \\ Key Laboratory of CNPC Geophysical Prospecting, China University of Petroleum, Beijing 102249, China
}

\begin{abstract}
In this paper, we analyze the seismic signal in the time-frequency domain using the generalized S-transform combined with spectrum modeling. Without assuming that the reflection coefficients are random white noise as in the conventional resolution-enhanced techniques, the wavelet which changes with time and frequency was simulated and eliminated. After using the inverse S-transform for the processed instantaneous spectrum, the signal in the time domain was obtained again with a more balanced spectrum and broader frequency band. The quality of seismic data was improved without additional noise.
\end{abstract}

Key words: Time-frequency domain, generalized S-transform, spectrum modeling, instantaneous spectrum, balanced spectrum

\section{Introduction}

Conventional techniques for enhancing seismic resolution, such as deconvolution, usually assume that the reflection coefficients are white noise, so the autocorrelation of the wavelet is equivalent to the auto-correlation of the seismic record (Yu and Zhao, 2002). However, the reflection coefficient is not a random sequence because of the thin interbeds and the multi-period sedimentary cycles. Accordingly, it is inaccurate to broaden the spectrum of the seismic record as that of wavelet (Zhao et al, 1996), and it also cannot satisfy the technical requirements for the prospecting for thin interbeds and lithologic reservoirs.

This paper started from enhancing the resolution through eliminating the wavelet, and discussed the validity of spectrum modeling on wavelet simulation. Considering the time variant characteristics of the wavelet, it is more accurate and effective to use spectrum modeling deconvolution in the time-frequency domain after the generalized S-transform instead of spectrum modeling deconvolution in the onedimensional frequency domain. This analysis process agrees with the seismic propagation underground and the result provides high quality seismic data for accurate interpretation.

\section{Extraction and elimination of seismic wavelet}

The reflection coefficient section is the perfect high resolution section. The seismic section can be considered as the reflection coefficient section which is influenced by wavelet. There are many wavelet extraction techniques to reduce the effect of wavelet (Yun and Ding, 2005; Sun,

*Corresponding author. email: tianjh13579@eyou.com

Received October 28, 2008 2000a; Li et al, 2007; Li et al, 2005), which make the processed section approach the actual interfaces underground.

Spectrum modeling is an effective and stable technique. This method considers that the amplitude spectrum of the wavelet is smooth and that of the reflection coefficients is vibratory without the assumption of random reflection coefficients. The wavelet can be simulated approximately as a specific curve from the amplitude spectrum of the seismic record and then is eliminated. The residual part is considered to be the amplitude spectrum of the reflection coefficients (Sun, 2000b).

With regard to the wavelet, Ricker deduced the amplitude spectrum of the Ricker wavelet (Ricker, 1977):

$$
W(f)=\left(\frac{2}{\sqrt{\pi}}\right)\left(\frac{f^{2}}{f_{\mathrm{c}}^{2}}\right) \exp \left(-\frac{f^{2}}{f_{\mathrm{c}}^{2}}\right)
$$

Rosa tested and summarized the empirical mathematical expression of the amplitude spectrum of the seismic wavelet (Rosa and Ulrych, 1991):

$$
W(f)=|f|^{k} \exp \left(\sum_{n=0}^{N} a_{n} f^{n}\right)
$$

where $k$ is a constant, $a_{n}$ is the coefficient of the multinomial of $f$.

In this way, the amplitude spectrum of the wavelet is simulated by using the least-square fitting method based on the amplitude spectrum of seismic record (as shown in Fig. 1).

Within the analyzed frequency band, the amplitude spectrum of the seismic record divided by that of the wavelet is the amplitude spectrum of the reflection coefficient. Keeping the phase of the seismic record invariable, we 


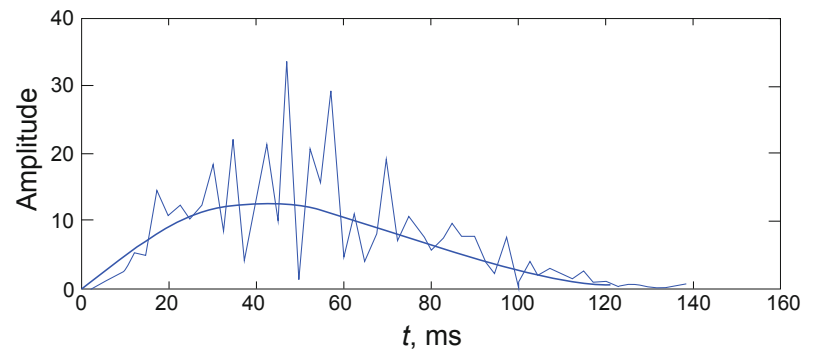

Fig. 1 Simulated smooth amplitude spectrum of the wavelet on the basis of the amplitude spectrum of the seismic record

performed the reverse FFT (Fast Fourier Transform) and obtained the record in the time domain.

Due to the time-variant characteristics of the seismic record, the amplitude at a specific frequency changes with time. Considering the absorption characteristics of rocks, the dominant frequency was reduced because of the propagation of the seismic wave (Fan and Zeng, 1995). Thus simulation in the frequency domain could not consider the instantaneous variation adequately (Fig. 1). We could analyze the seismic signal within the time window to overcome this deficiency. However, the frequency variation may be great between two adjacent layers, and the spectrum analysis in the time window which reflects the average effect of frequencies affects the processing result. It is necessary to transform the seismic record into the two-dimensional time-frequency domain, and the spectrum modeling may be more feasible and effective.

\section{Spectrum modeling in time-frequency domain}

\subsection{S-transform and generalized S-transform}

The S-transform of time series $h(t)$ is defined by:

$$
S(\tau, f)=\int_{-\infty}^{+\infty} h(t) \cdot \frac{|f|}{\sqrt{2 \pi}} \cdot \exp \left(-\frac{(\tau-t)^{2} f^{2}}{2}\right) \cdot \exp (-i 2 \pi f t) \mathrm{d} t
$$

where $f$ is frequency, and $\tau$ is the midpoint of the time window, thus it also denotes the location of the window function in the time axis.

The S-transform is the combination and improvement of the short time Fourier Transform and the continuous wavelet transform (Stockwell et al, 1996). It has the characteristics of frequency-dependent resolution which the short time Fourier Transform does not have. It indeed provides the global signal in the time-frequency domain compared with the time-scale domain analysis of the continuous wavelet transform.

The inverse S-transform is defined by:

$$
h(t)=\int_{-\infty}^{+\infty}\left(\int_{-\infty}^{+\infty} S(\tau, f) \mathrm{d} \tau\right) \exp (i 2 \pi f t) \mathrm{d} f
$$

One problem of the S-transform in practical application is the fixed Gaussian window, and the generalized S-transform (GST) is designed to resolve this problem (Pinnegar and
Mansinha, 2003). Gao summarized the previous expressions, and the basis function is normalized as follows (Gao et al, 2003):

$$
g_{(f)}(t) \exp \left(-i 2 \pi f_{0} f t\right)=A|f| \exp \left(-\alpha(f t-\beta)^{2}-i 2 \pi f_{0} f t\right)
$$

where $A$ is the amplitude of the basic wavelet, $\alpha$ is the attenuation rate of the energy, $\beta$ is the time-lag factor of energy, $f_{0}$ is the apparent frequency of the basic wavelet. The GST provides better time and frequency resolution than the S-transform while maintaining the advantages of the S-transform.

The solid line in Fig. 2(a) is a Ricker wavelet whose dominant frequency is $30 \mathrm{~Hz}$, and sampling interval is $1 \mathrm{~ms}$. Fig. 2(b) is the signal after inverse S-transform. Fig. 2(c) shows the error curve and the average absolute error is $3.16 \times$ $10^{-5}$. We can draw a conclusion that the GST can reconstruct the signal without loss. This provides the feasibility of processing signal in time-frequency domain and then transforming back to time domain.

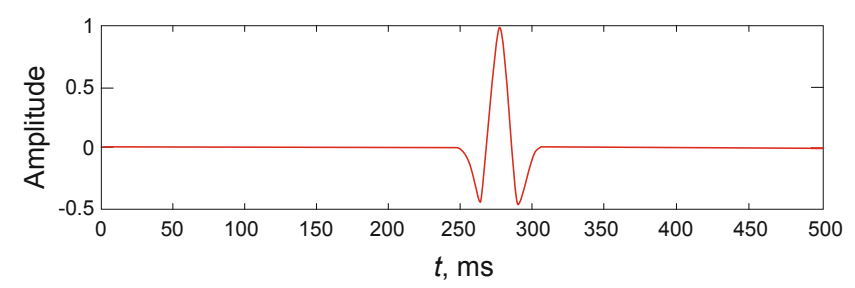

(a) Ricker wavelet

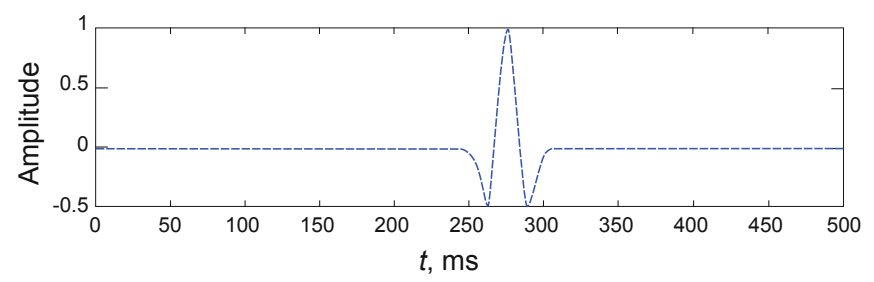

(b) Signal after inverse generalized S-transform

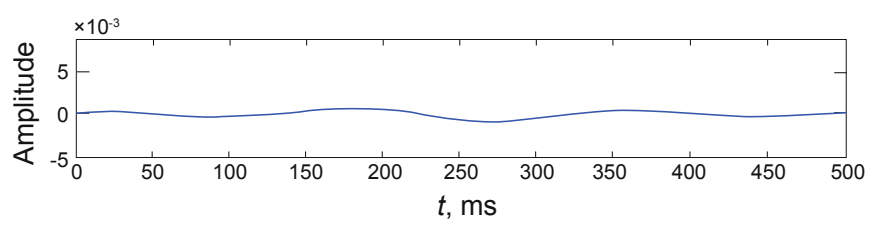

(c) Error curve

Fig. 2 Characteristics of reconstruction without loss of GST

\subsection{Wavelet spectrum in time-frequency domain based on the generalized S-transform}

GST is a perfect time-frequency analysis tool, and we can simulate the wavelet spectrum which changes with time and frequency.

Fig. 3(a) shows a seismic reflection sequence from a thin interbedded model. Fig. 3(b) is the seismic response using a Ricker wavelet whose primary dominant frequency is $30 \mathrm{~Hz}$. Fig. 3(c) is the 3D plot of the wavelet changing with time and frequency, which shows specific movements in time-frequency domain. Fig. 3(d) shows the signal after 


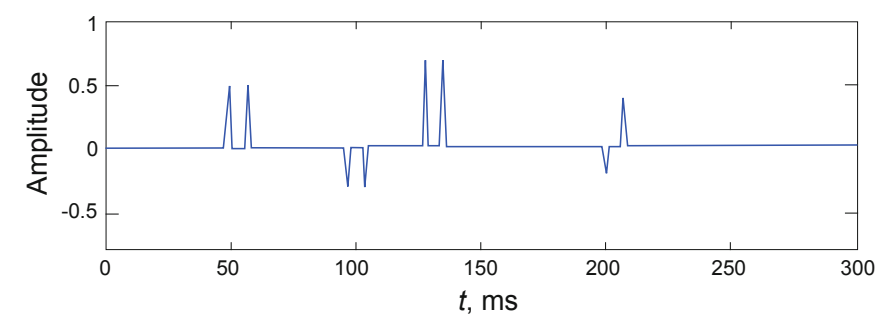

(a) Reflection sequence model

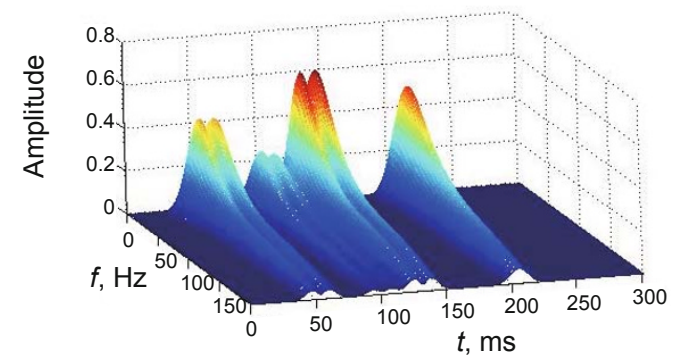

(c) 3D time-variant wavelet spectrum

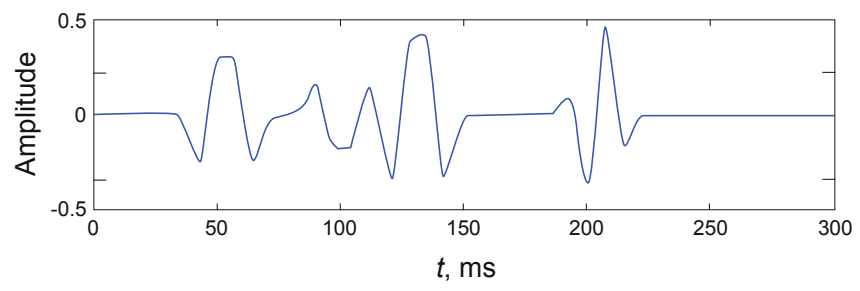

(b) Synthetic seismic record

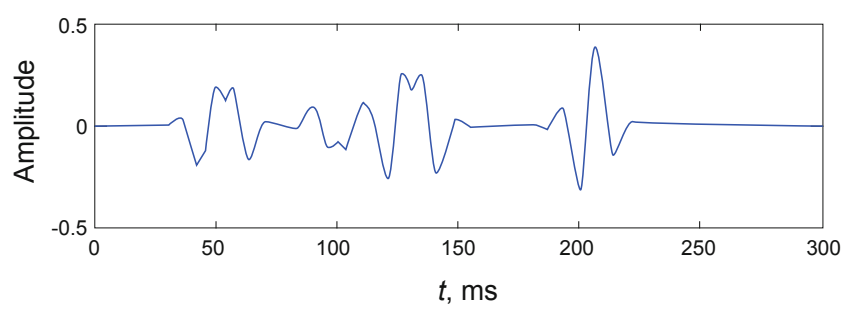

(d) Seismic record after deconvolution

Fig. 3 Model experiment of spectrum modeling in the time-frequency domain

inverse S-transform. We can see that the adjacent events are distinguished, and the shape of signal after processing is closer to the reflection sequence.

\section{Actual seismic data processing}

Fig. 4(a) shows a post-stack seismic section. Fig. 4(b) is the result after processing by spectral whitening. Fig. 4(c) is the section after deconvolution in the time-frequency domain by using GST. Compared with Fig. 4(a), both Fig. 4(b) and Fig. 4(c) improve the resolution but Fig. 4(c) reveals more details than Fig. 4(b).

Because of the plentiful frequency components in the actual seismic data, it is necessary to analyze the seismic record in the time-frequency domain before spectrum modeling. The distribution of amplitude in the time-frequency domain reflects the characteristics of the sedimentary cycle of formation (Wang et al, 2008), thus we should consider the geological significance and choose reasonable instantaneous frequency bands, within which we can simulate the seismic wavelet (Liu et al, 2006a; 2006b). Fig. 4(d) shows one seismic trace and the wavelet distribution in the timefrequency domain. Fig. 4(e) is the comparison of spectrum analysis, from which we can see that the processed result has a broader frequency band and higher dominant frequency.

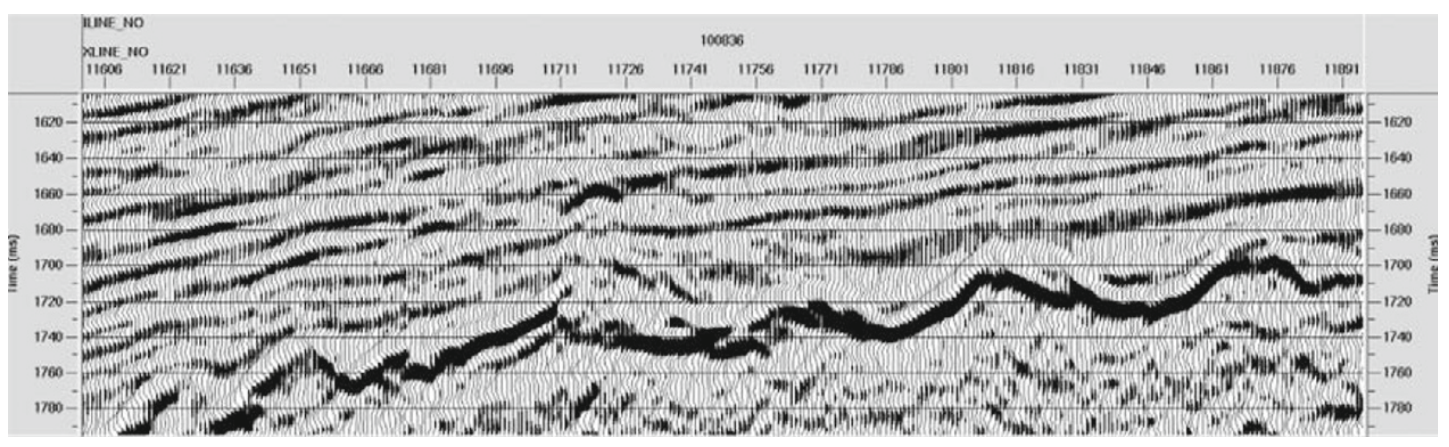

(a) Seismic section before processing

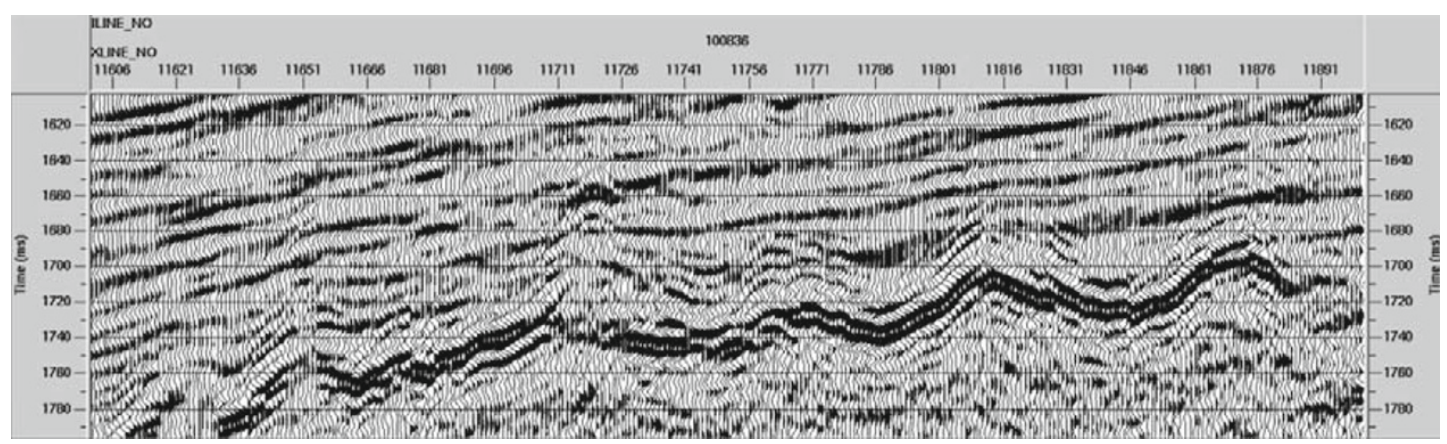

(b) Seismic section processed by conventional method 


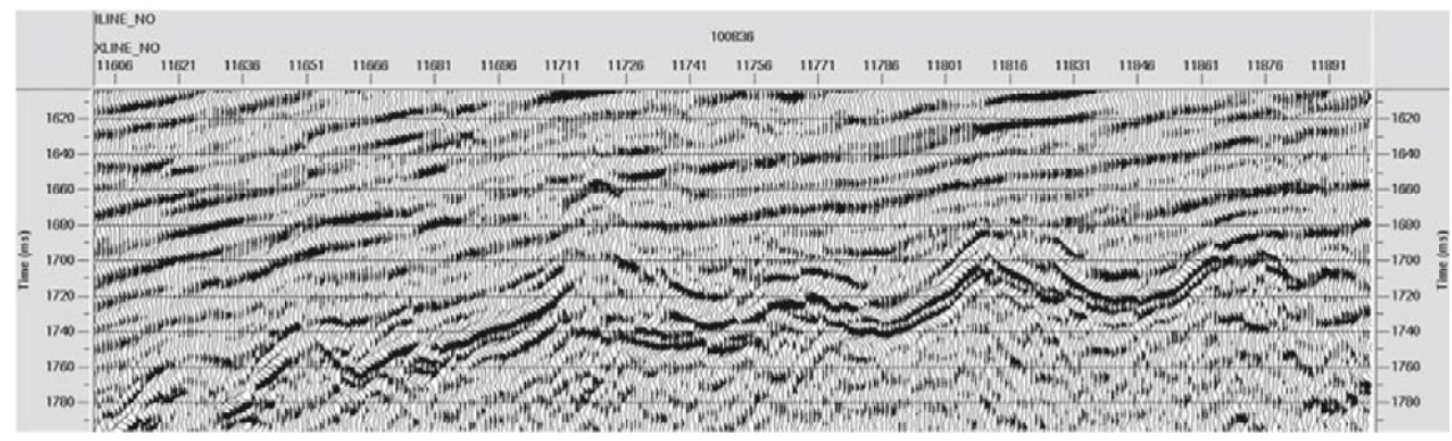

(c) Seismic section processed by our technique
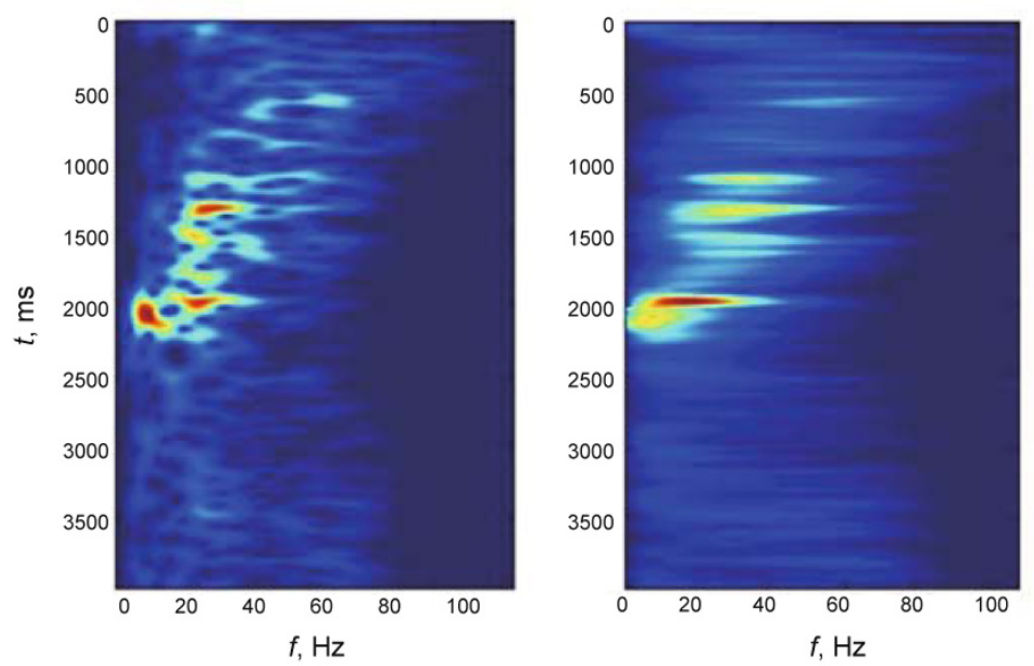

(d) Time-frequency analysis of one seismic trace and the simulated instantaneous spectrum of the wavelet

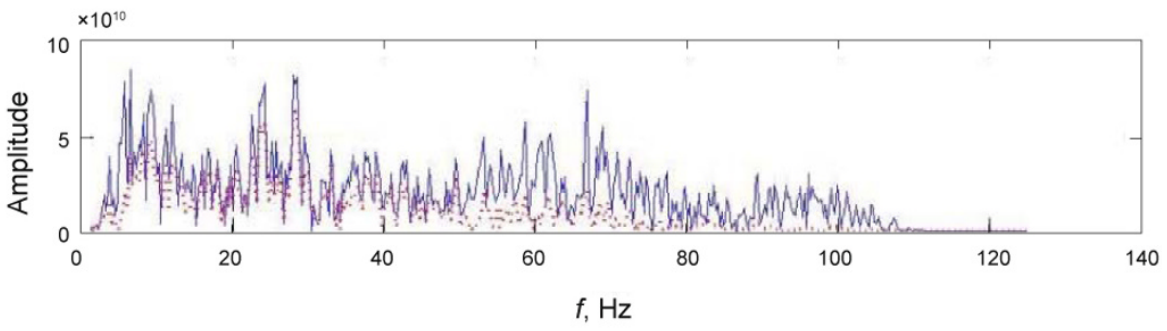

(e) Comparison of spectrum analysis. Dashed line is the spectrum before processing, and solid line is the spectrum after processing

Fig. 4 Actual seismic data processing and analysis

\section{Conclusions}

1) We obtained the amplitude distribution of the seismic record in the time-frequency domain using the generalized S-transform, and the wavelets changing with time were simulated. Compared with spectrum modeling in the frequency domain, our technique is more flexible and accurate.

2) The frequency bands chosen from the time-frequency distribution by considering geological significance were appropriate. The result using our technique revealed more details in the seismic section than conventional high resolution processing methods. We could obtain more specific seismic data for the following work, such as interpretation and inversion.

\section{Acknowledgements}

This research was financially supported by National 973 Key Basic Research Development Program (N0.2007CB209602) and National 863 High Technology Research Development Program (No.2007AA06Z229). 


\section{References}

Fan X D and Zeng H. Time-space variant spectrum whitening of seismic data. Oil Geophysical Prospecting. 1995. 30(4): 550-555 (in Chinese)

Gao J H, Chen W C, Li Y M, et al. Generalized S transform and seismic response analysis of thin interbeds. Geophysics. 2003. 46(4): 526532

Li D W, Yin C, Zhao W K, et al. Seismic wavelet extraction based on phase scan. Journal of Southwest Petroleum University. 2007. 29(3): 17-19 (in Chinese)

Li G F, Peng S P, Gao R S, et al. Method of mixed phase wavelet extraction in spectrum domain. Natural Gas Industry. 2005. 25(1): 8587 (in Chinese)

Liu X W, Liu H, Li Y M, et al. Study on characteristics of seismic stratigraphy by generalized S-transform. Progress in Geophysics. 2006. 21(2): 440-451 (in Chinese)

Liu X W, Nian J B and Liu H. Generalized S-transform based seismic attenuation analysis. Progress in Geophysics. 2006. 29(1): 20-24 (in Chinese)

Pinnegar C R and Mansinha L. The S-transform with windows of arbitrary and varying shape. Geophysics. 2003. 68(1): 381-385

Ricker N H. Transient Waves in Visco-elastic Media. Amsterdam: Elsevier Science \& Technology Publishing Company. 1977
Rosa A L and Ulrych T J. Processing via spectral modeling. Geophysics. 1991. 56(8): 1244-1251

Stockwell R G, Mansinha L and Lowe R P. Localization of the complex spectrum: the $\mathrm{S}$ transform. IEEE Transactions on Signal Processing. 1996. 44(4): 998-1001

Sun C Y. Study on the extraction of spatial varied seismic wavelets. Journal of China University of Petroleum (Edition of Natural Science). 2000a. 24(1): 77-84 (in Chinese)

Sun C Y. Spectrum modeling method and its application to seismic resolution improvement. Oil Geophysical Prospecting. 2000b. 35(1): 27-35 (in Chinese)

Wang P, Li G F, Zhang L Q, et al. Thin-layer reservoir prediction in the lower Es_1 of the western ramp of Qikou Depression. Journal of China University of Petroleum (Edition of Natural Science). 2008. 32(2): 28-33 (in Chinese)

Yun M H and Ding W. Analysis of seismic wavelet frequency. Geophysical Prospecting for Petroleum. 2005. 44(6): 578-581 (in Chinese)

Yu P and Zhao Z Y. Three techniques of deconvolution in seismic data processing. Global Geology. 2002. 21(2): 181-184 (in Chinese)

Zhao B, Yu S P, Nie X B, et al. Spectral-modeled deconvolution and its application. Oil Geophysical Prospecting. 1996. 31(1): 101-116 (in Chinese)

(Edited by Hao Jie) 